Bull. Soc. math. France

133 (2), 2005, p. 259-273

\title{
ESTIMATES OF THE KOBAYASHI-ROYDEN METRIC IN ALMOST COMPLEX MANIFOLDS
}

\author{
By Hervé Gaussier \& Alexandre Sukhov
}

\begin{abstract}
We establish a lower estimate for the Kobayashi-Royden infinitesimal pseudometric on an almost complex manifold $(M, J)$ admitting a bounded strictly plurisubharmonic function. We apply this result to study the boundary behaviour of the metric on a strictly pseudoconvex domain in $M$ and to give a sufficient condition for the complete hyperbolicity of a domain in $(M, J)$.

RÉSUMÉ (Estimées de la métrique de Kobayashi-Royden dans les variétés presque complexes)

Nous établissons une estimée inférieure pour la métrique de Kobayashi-Royden sur une variété presque complexe $(M, J)$ admettant une fonction bornée strictement pluri-sous-harmonique. Nous appliquons ce résultat à l'étude du comportement de la métrique au bord d'un domaine strictement pseudoconvexe dans $M$ et donnons une condition suffisante d'hyperbolicité complète d'un domaine dans $(M, J)$.
\end{abstract}

\section{Introduction}

In the recent paper [11], S. Kobayashi studied the following question: Does every point in an almost complex manifold admit a basis of complete hyperbolic

Texte reçu le 25 mars 2003, révisé le 18 décembre 2003, accepté le 6 mars 2004

Hervé Gaussier, LATP UMR 6632, CMI, 39 rue Joliot-Curie, 13453 Marseille Cedex 13

(France). - E-mail : gaussier@cmi.univ-mrs.fr • Url : www.cmi.univ-mrs.fr Alexandre Sukhov, AGAT UMR 8524, USTL, Cité Scientifique, 59655 Villeneuve d'Ascq Cedex (France). • E-mail : sukhov@agat.univ-lille1.fr • Url : www-gat.univlille1.fr

2000 Mathematics Subject Classification. - 32V40, 32V15, 32H40, 32T15, 53C15.

Key words and phrases. - Almost complex manifolds, Kobayashi-Royden metric, $J$-holomorphic discs.

BULletiN DE LA SOCIÉTÉ MATHÉMATIQUE DE FRANCE

(C) Société Mathématique de France

$0037-9484 / 2005 / 259 / \$ 5.00$ 
neighborhoods? This question was solved in dimension 4 by R. Debalme and S. Ivashkovich in [5].

In the present paper, we give a lower estimate on the Kobayashi-Royden infinitesimal metric on a strictly pseudoconvex domain in an almost complex manifold (such estimates are well-known in the integrable case [7]). A corollary of our main result gives a positive answer to the previous question in any dimension: every point in an almost complex manifold has a complete hyperbolic neighborhood.

Our approach consists of two parts. In order to localize the KobayashiRoyden metric we use a method developped essentially by N. Sibony [15] in the case of the standard complex structure (see also [6] by K. DiederichJ.E. Fornaess and [10] by N. Kerzman \& J.-P. Rosay). This is based on the construction of special classes of plurisubharmonic functions. Then we apply an almost complex analogue of the scaling method due to S. Pinchuk in the integrable case (see, for instance, [14]) and obtain presice estimates of the metric. We point out that similar ideas have been used by F. Berteloot [1], [2] in order to estimate the Kobayashi-Royden metric on some classes of domains in $\mathbb{C}^{n}$.

We note that S. Ivashkovich and J.-P. Rosay recently proved in [9], among other results, estimates of the Kobayashi-Royden metric similar to ours under weaker assumptions on the regularity of the almost complex structure.

Acknowledgments. - The authors thank E. Chirka, B. Coupet, S. Ivashkovich and J.-P. Rosay for helpful discussions and the referee for valuable remarks. We are particularly indebted to S. Ivashkovich who pointed out an erroneous argument in the previous version of our paper.

\section{Preliminaries}

1.1. Almost complex manifolds. - Let $\left(M^{\prime}, J^{\prime}\right)$ and $(M, J)$ be almost complex manifolds and let $f$ be a smooth map from $M^{\prime}$ to $M$. We say that $f$ is $\left(J^{\prime}, J\right)$-holomorphic if $\mathrm{d} f \circ J^{\prime}=J \circ \mathrm{d} f$ on $T M^{\prime}$. We denote by $\mathcal{O}_{\left(J^{\prime}, J\right)}\left(M^{\prime}, M\right)$ the set of $\left(J^{\prime}, J\right)$-holomorphic maps from $M^{\prime}$ to $M$. Let $\Delta$ be the unit disc in $\mathbb{C}$ and $J_{\text {st }}$ be the standard integrable structure on $\mathbb{C}^{n}$ for every $n$. If $\left(M^{\prime}, J^{\prime}\right)=\left(\Delta, J_{\text {st }}\right)$, we denote by $\mathcal{O}_{J}(\Delta, M)$ the set $\mathcal{O}_{\left(J_{\mathrm{st}}, J\right)}(\Delta, M)$ of $J$-holomorphic discs in $M$.

The following lemma shows that every almost complex manifold $(M, J)$ can be viewed locally as the unit ball in $\mathbb{C}^{n}$ equipped with a small almost complex deformation of $J_{\text {st }}$. This will be used frequently in the sequel.

Lemma 1. - Let $(M, J)$ be an almost complex manifold. Then for every point $p \in M$ and every $\lambda_{0}>0$ there exist a neighborhood $U$ of $p$ and a coordinate diffeomorphism $z: U \rightarrow \mathbb{B}$ such that $z(p)=0, \mathrm{~d} z(p) \circ J(p) \circ \mathrm{d} z^{-1}(0)=J_{\text {st }}$ and the direct image $z_{*}(J):=\mathrm{d} z \circ J \circ \mathrm{d} z^{-1}$ satisfies $\left\|z_{*}(J)-J_{\mathrm{st}}\right\|_{\mathcal{C}^{2}(\overline{\mathbb{B}})} \leq \lambda_{0}$. 
Proof. - There exists a diffeomorphism $z$ from a neighborhood $U^{\prime}$ of $p \in M$ onto $\mathbb{B}$ satisfying $z(p)=0$ and $\mathrm{d} z(p) \circ J(p) \circ \mathrm{d} z^{-1}(0)=J_{\text {st }}$. For $\lambda>0$ consider the dilation $\mathrm{d}_{\lambda}: t \mapsto \lambda^{-1} t$ in $\mathbb{C}^{n}$ and the composition $z_{\lambda}=\mathrm{d}_{\lambda} \circ z$. Then $\lim _{\lambda \rightarrow 0}\left\|\left(z_{\lambda}\right)_{*}(J)-J_{\text {st }}\right\|_{\mathcal{C}^{2}(\overline{\mathbb{B}})}=0$. Setting $U=z_{\lambda}^{-1}(\mathbb{B})$ for $\lambda>0$ small enough, we obtain the desired statement.

1.2. The operators $\partial_{J}$ and $\bar{\partial}_{J}$. - Let $(M, J)$ be an almost complex manifold. We denote by $T M$ the real tangent bundle of $M$ and by $T_{\mathbb{C}} M$ its complexification. Recall that $T_{\mathbb{C}} M=T^{(1,0)} M \oplus T^{(0,1)} M$ where

$$
\begin{aligned}
T^{(1,0)} M & :=\left\{X \in T_{\mathbb{C}} M ; J X=i X\right\}=\{\zeta-i J \zeta ; \zeta \in T M\}, \\
T^{(0,1)} M & :=\left\{X \in T_{\mathbb{C}} M ; J X=-i X\right\}=\{\zeta+i J \zeta ; \zeta \in T M\} .
\end{aligned}
$$

Let $T^{*} M$ denote the cotangent bundle of $M$. Identifying $\mathbb{C} \otimes T^{*} M$ with $T_{\mathbb{C}}^{*} M:=\operatorname{Hom}\left(T_{\mathbb{C}} M, \mathbb{C}\right)$ we define the set of complex forms of type $(1,0)$ on $M$ by

$$
T_{(1,0)} M=\left\{w \in T_{\mathbb{C}}^{*} M ; w(X)=0, \forall X \in T^{(0,1)} M\right\}
$$

and the set of complex forms of type $(0,1)$ on $M$ by

$$
T_{(0,1)} M=\left\{w \in T_{\mathbb{C}}^{*} M ; w(X)=0, \forall X \in T^{(1,0)} M\right\} .
$$

Then $T_{\mathbb{C}}^{*} M=T_{(1,0)} M \oplus T_{(0,1)} M$. This allows to define the operators $\partial_{J}$ and $\bar{\partial}_{J}$ on the space of smooth functions defined on $M$ : given a complex smooth function $u$ on $M$, we set $\partial_{J} u=\mathrm{d} u_{(1,0)} \in T_{(1,0)} M$ and $\bar{\partial}_{J} u=\mathrm{d} u_{(0,1)} \in T_{(0,1)} M$. As usual, differential forms of any bidegree $(p, q)$ on $(M, J)$ are defined by means of the exterior product.

\section{2. $J$-plurisubharmonic functions with logarithmic singularities}

2.1. Plurisubharmonic functions. - We first recall the following definition:

Definition 1. - An upper semicontinuous function $u$ on $(M, J)$ is called $J$ plurisubharmonic on $M$ if the composition $u \circ f$ is subharmonic on $\Delta$ for every $f \in \mathcal{O}_{J}(\Delta, M)$.

If $M$ is a domain in $\mathbb{C}^{n}$ and $J=J_{\text {st }}$ then a $J_{\text {st }}$-plurisubharmonic function is a plurisubharmonic function in the usual sense.

Definition 2. - Let $u$ be a $\mathcal{C}^{2}$ function on $M$, let $p \in M$ and $v \in T_{p} M$. The Levi form of $u$ at $p$, evaluated on $v$, is defined by

$$
\mathcal{L}^{J}(u)(p)(v):=-\mathrm{d}\left(J^{\star} \mathrm{d} u\right)(X, J X)(p),
$$

where $X$ is any vector field on $T M$ such that $X(p)=v$.

Following [4], [8] we have:

BULlETIN DE LA SOCIÉTÉ MATHÉMATIQUE DE FRANCE 
Proposition 1. - Let $u$ be a $\mathcal{C}^{2}$ real valued function on $M$, let $p \in M$ and $v \in T_{p} M$. Then $\mathcal{L}^{J}(u)(p)(v)=\Delta(u \circ f)(0)$ where $f$ is any $J$-holomorphic disc in $M$ satisfying $f(0)=p, \mathrm{~d} f(0)(\partial / \partial x)=v$.

Obviously the Levi form is invariant with respect to biholomorphisms. More precisely let $u$ be a $\mathcal{C}^{2}$ real valued function on $M$, let $p \in M$ and $v \in T_{p} M$. If $\Phi$ is a diffeomorphism from $(M, J)$ to $\left(M^{\prime}, J^{\prime}\right),\left(J, J^{\prime}\right)$-holomorphic, then

$$
\mathcal{L}^{J}(u)(p)(v)=\mathcal{L}^{J^{\prime}}\left(u \circ \Phi^{-1}\right)(\Phi(p))(\mathrm{d} \Phi(p)(v)) .
$$

Finally it follows from Proposition 1 that a $\mathcal{C}^{2}$ real valued function $u$ on $M$ is $J$-plurisubharmonic on $M$ if and only if $\mathcal{L}^{J}(u)(p)(v) \geq 0$ for every $p \in M$, $v \in T_{p} M$. This leads to the definition:

Definition 3. - A $\mathcal{C}^{2}$ real valued function $u$ on $M$ is strictly $J$-plurisubharmonic on $M$ if $\mathcal{L}^{J}(u)(p)(v)$ is positive for every $p \in M, v \in T_{p} M \backslash\{0\}$.

We have the following example of a $J$-plurisubharmonic function on an almost complex manifold $(M, J)$ :

EXAMPLE 1. - For every point $p \in(M, J)$ there exists a neighborhood $U$ of $p$ and a diffeomorphism $z: U \rightarrow \mathbb{B}$ centered at $p$ (i.e. $z(p)=0$ ) such that the function $|z|^{2}$ is $J$-plurisubharmonic on $U$.

Proof. - Let $p \in M, U_{0}$ be a neighborhood of $p$ and $z: U_{0} \rightarrow \mathbb{B}$ be local complex coordinates centered at $p$, such that $\mathrm{d} z \circ J(p) \circ \mathrm{d} z^{-1}=J_{\text {st }}$ on $\mathbb{B}$. Consider the function $u(q)=|z(q)|^{2}$ on $U_{0}$. For every $w, v \in \mathbb{C}^{n}$ we have $\mathcal{L}^{J_{\mathrm{st}}}\left(u \circ z^{-1}\right)(w)(v)=\|v\|^{2}$. Let $B\left(0, \frac{1}{2}\right)$ be the ball centered at the origin with radius $\frac{1}{2}$ and let $\mathcal{E}$ be the space of smooth almost complex structures defined in a neighborhood of $\overline{B\left(0, \frac{1}{2}\right)}$. Since the function $\left(J^{\prime}, w\right) \mapsto \mathcal{L}^{J^{\prime}}\left(u \circ z^{-1}\right)(w)$ is continuous on $\mathcal{E} \times B\left(0, \frac{1}{2}\right)$, there exist a neighborhood $V$ of the origin and positive constants $\lambda_{0}$ and $c$ such that $\mathcal{L}^{J^{\prime}}\left(u \circ z^{-1}\right)(q)(v) \geq c\|v\|^{2}$ for every $q \in V$ and for every almost complex structure $J^{\prime}$ satisfying $\left\|J^{\prime}-J_{\mathrm{st}}\right\|_{\mathcal{C}^{2}(\bar{V})} \leq \lambda_{0}$. Let $U_{1}$ be a neighborhood of $p$ such that

$$
\left\|z_{*}(J)-J_{\mathrm{st}}\right\|_{\mathcal{C}^{2}\left(\overline{z\left(U_{1}\right)}\right)} \leq \lambda_{0}
$$

and let $0<r<1$ be such that $B(0, r) \subset V$ and $U:=z^{-1}(B(0, r)) \subset U_{1}$. Then we have the following estimate for every $q \in U$ and $v \in T_{q} M$ :

$$
\mathcal{L}^{J}(u)(q)(v) \geq c\|v\|^{2} .
$$

Then $r^{-1} z$ is the desired diffeomorphism.

We also have the following 
Lemma 2. - A function $u$ of class $\mathcal{C}^{2}$ in a neighborhood of a point $p$ of $(M, J)$ is strictly $J$-plurisubharmonic if and only there exists a neighborhood $U$ of $p$ with local complex coordinates $z: U \rightarrow \mathbb{B}$ centered at $p$, such that the function $u-c|z|^{2}$ is J-plurisubharmonic on $U$ for some constant $c>0$.

The function $\log |z|$ is $J_{\text {st }}$-plurisubharmonic on $\mathbb{C}^{n}$ and plays an important role in the pluripotential theory as the Green function for the complex MongeAmpère operator on the unit ball. In particular, this function is crucially used in Sibony's method in order to localize and estimate the Kobayashi-Royden metric on a complex manifold. Unfortunately, after an arbirarily small general almost complex deformation of the standard structure this function is not plurisubharmonic with respect to the new structure (in any neighborhood of the origin), see for instance [4]. So we will need the following statement communicated to the authors by E. Chirka:

Lemma 3. - Let $p$ be a point in an almost complex manifold $(M, J)$. There exist a neighborhood $U$ of $p$ in $M$, a diffeomorphism $z: U \rightarrow \mathbb{B}$ centered at $p$ and positive constants $\lambda_{0}, A$, such that $\log |z|+A|z|$ is $J^{\prime}$-plurisubharmonic on $U$ for every almost complex structure $J^{\prime}$ satisfying $\left\|J^{\prime}-J\right\|_{\mathcal{C}^{2}(\bar{U})} \leq \lambda_{0}$.

Proof. - Consider the function $u=|z|$ on $\mathbb{B}$. Since

$$
\mathcal{L}^{J_{\mathrm{st}}}\left(u \circ z^{-1}\right)(w)(v) \geq \frac{\|v\|^{2}}{4|w|}
$$

for every $w \in \mathbb{B} \backslash\{0\}$ and every $v \in \mathbb{C}^{n}$, it follows by a direct expansion of $\mathcal{L}^{J^{\prime}}(u)$ that there exist a neighborhood $U$ of $p, U \subset \subset U_{0}$, and a positive constant $\lambda_{0}$ such that $\mathcal{L}^{J^{\prime}}(u)(q)(v) \geq\|v\|^{2} / 5|z(q)|$ for every $q \in U \backslash\{p\}$, every $v \in T_{q} M$ and every almost complex structure $J^{\prime}$ satisfying $\left\|J^{\prime}-J\right\|_{\mathcal{C}^{2}(\bar{U})} \leq \lambda_{0}$. Moreover, computing the Laplacian of $\log |f|$ where $f$ is any $J$-holomorphic disc we obtain, decreasing $\lambda_{0}$ if necessary, that there exists a positive constant $B$ such that $\mathcal{L}^{J^{\prime}}(\log |z|)(q)(v) \geq-B\|v\|^{2} /|z(q)|$ for every $q \in U \backslash\{p\}$, every $v \in T_{q} M$ and every almost complex structure $J^{\prime}$ satisfying $\left\|J^{\prime}-J\right\|_{\mathcal{C}^{2}(\bar{U})} \leq \lambda_{0}$. We may choose $A=2 B$ to get the result.

\section{Localization of the Kobayashi-Royden metric on almost complex manifolds}

Let $(M, J)$ be an almost complex manifold. In what follows we use the notation $\zeta=x+i y \in \mathbb{C}$. According to [13], for every $p \in M$ there is a neighborhood $\mathcal{V}$ of 0 in $T_{p} M$ such that for every $v \in \mathcal{V}$ there exists $f \in \mathcal{O}_{J}(\Delta, M)$ satisfying $f(0)=p, \mathrm{~d} f(0)(\partial / \partial x)=v$. This allows to define the KobayashiRoyden infinitesimal pseudometric $K_{(M, J)}$.

BULlETIN DE LA SOCIÉTÉ MATHÉMATIQUE DE FRANCE 
Definition 4. - For $p \in M$ and $v \in T_{p} M, K_{(M, J)}(p, v)$ is the infimum of the set of positive $\alpha$ such that there exists a $J$-holomorphic disc $f: \Delta \rightarrow M$ satisfying $f(0)=p$ and $\mathrm{d} f(0)(\partial / \partial x)=v / \alpha$.

Since for every $f \in \mathcal{O}_{\left(J^{\prime}, J\right)}\left(M^{\prime}, M\right)$ and every $\varphi \in \mathcal{O}_{J}\left(\Delta, M^{\prime}\right)$ the composition $f \circ \varphi$ is in $\mathcal{O}_{J}(\Delta, M)$ we have:

Proposition 2. - Let $f:\left(M^{\prime}, J^{\prime}\right) \rightarrow(M, J)$ be a $\left(J^{\prime}, J\right)$-holomorphic map. Then for every $p^{\prime} \in M^{\prime}$ and $v^{\prime} \in T_{p^{\prime}} M^{\prime}$,

$$
K_{(M, J)}\left(f\left(p^{\prime}\right), \mathrm{d} f\left(p^{\prime}\right)\left(v^{\prime}\right)\right) \leq K_{\left(M^{\prime}, J^{\prime}\right)}\left(p^{\prime}, v^{\prime}\right) .
$$

We denote by $d_{(M, J)}^{K}$ the integrated pseudodistance of the KobayashiRoyden infinitesimal pseudometric. According to the almost complex version of Royden's theorem [12], it coincides with the usual Kobayashi pseudodistance on $(M, J)$ defined by means of $J$-holomorphic discs. Similarly to the case of the integrable structure we have:

Definition 5. - (i) Let $p \in M$. Then $M$ is locally hyperbolic at $p$ if there exists a neighborhood $U$ of $p$ and a positive constant $C$ such that for every $q \in U, v \in T_{q} M: K_{(M, J)}(q, v) \geq C\|v\|$.

(ii) $(M, J)$ is hyperbolic if it is locally hyperbolic at every point.

(iii) $(M, J)$ is complete hyperbolic if the Kobayashi ball

$$
B_{(M, J)}^{K}(p, r):=\left\{q \in M ; d_{(M, J)}^{K}(p, q)<r\right\}
$$

is relatively compact in $M$ for every $p \in M, r \geq 0$.

LEMMA 4. - Let $r<1$ and let $\theta_{r}$ be a smooth nondecreasing function on $\mathbb{R}^{+}$ such that $\theta_{r}(s)=s$ for $s \leq \frac{1}{3} r$ and $\theta_{r}(s)=1$ for $s \geq \frac{2}{3} r$. Let $(M, J)$ be an almost complex manifold, and let $p$ be a point of $M$. Then there exists a neighborhood $U$ of $p$, positive constants $A=A(r), B=B(r)$ and a diffeomorphism $z: U \rightarrow \mathbb{B}$ such that $z(p)=0, \mathrm{~d} z(p) \circ J(p) \circ \mathrm{d} z^{-1}(0)=J_{0}$ and the function $\log \left(\theta_{r}\left(|z|^{2}\right)\right)+\theta_{r}(A|z|)+B|z|^{2}$ is J-plurisubharmonic on $U$.

Proof of Lemma 4. - Denote by $w$ the standard coordinates in $\mathbb{C}^{n}$. It follows from Lemma 3 that there exist positive constants $A$ and $\lambda_{0}$ such that the function $\log \left(|w|^{2}\right)+A|w|$ is $J^{\prime}$-plurisubharmonic on $\mathbb{B}$ for every almost complex structure $J^{\prime}$, defined in a neighborhood of $\overline{\mathbb{B}}$ in $\mathbb{C}^{n}$ and such that $\left\|J^{\prime}-J_{\mathrm{st}}\right\|_{\mathcal{C}^{2}(\overline{\mathbb{B}})} \leq \lambda_{0}$. This means that the function

$$
v(w)=\log \left(\theta_{r}\left(|w|^{2}\right)\right)+\theta_{r}(A|w|)
$$

is $J^{\prime}$-plurisubharmonic on $B\left(0, r^{\prime}\right)=\left\{w \in \mathbb{C}^{n} ;|w|<r^{\prime}\right\}$ for every such almost complex structure $J^{\prime}$, where $r^{\prime}=\inf (\sqrt{r / 3}, r / 3 A)$. Decreasing $\lambda_{0}$ if necessary, we may assume that the function $|w|^{2}$ is strictly $J^{\prime}$-plurisubharmonic on $\mathbb{B}$. Then, since $v$ is smooth on $\mathbb{B} \backslash B\left(0, r^{\prime}\right)$, there exists a positive constant $B$ such that the function $v+B|w|^{2}$ is $J^{\prime}$-plurisubharmonic on $\mathbb{B}$ for $\left\|J^{\prime}-J_{\mathrm{st}}\right\|_{\mathcal{C}^{2}(\overline{\mathbb{B}})} \leq \lambda_{0}$. 
According to Lemma 1 there exists a neighborhood $U$ of $p$ and a diffeomorphism $z: U \rightarrow \mathbb{B}$ such that $\left\|z_{*}(J)-J_{\mathrm{st}^{t}}\right\|_{\mathcal{C}^{2}(\overline{\mathbb{B}})} \leq \lambda_{0}$. Then the function $v \circ z=$ $\log \left(\theta_{r}\left(|z|^{2}\right)\right)+\theta_{r}(A|z|)+B|z|^{2}$ is $J$-plurisubharmonic on $U$.

Proposition 3 (Localization principle). - Let $D$ be a domain in an almost complex manifold $(M, J)$, let $p \in \bar{D}$, let $U$ be a neighborhood of $p$ in $M$ (not necessarily contained in $D$ ) and let $z: U \rightarrow \mathbb{B}$ be the diffeomorphism given by Lemma 4 . Let $u$ be a $\mathcal{C}^{2}$ function on $\bar{D}$, negative and $J$-plurisubharmonic on $D$. We assume that $-L \leq u<0$ on $D \cap U$ and that $u-c|z|^{2}$ is $J$-plurisubharmonic on $D \cap U$, where $c$ and $L$ are positive constants. Then there exist a positive constant $s$ and a neighborhood $V \subset \subset U$ of $p$, depending on $c$ and $L$ only, such that for $q \in D \cap V$ and $v \in T_{q} M$ we have the following inequality:

$$
K_{(D, J)}(q, v) \geq s K_{(D \cap U, J)}(q, v) .
$$

We note that a similar statement was obtained by F. Berteloot [1] in the integrable case. The proof is based on N. Sibony's method [15].

Proof of Proposition 3. - Let $0<r<1$ be such that the set $V_{1}:=$ $\{q \in U ;|z(q)| \leq \sqrt{r}\}$ is relatively compact in $U$ and let $\theta_{r}$ be a smooth nondecreasing function on $\mathbb{R}^{+}$such that $\theta_{r}(s)=s$ for $s \leq \frac{1}{3} r$ and $\theta_{r}(s)=1$ for $s \geq \frac{2}{3} 2 r$. According to Lemma 4, there exist uniform positive constants $A$ and $B$ such that the function

$$
\log \left(\theta_{r}\left(|z-z(q)|^{2}\right)\right)+\theta_{r}(A|z-z(q)|)+B|z|^{2}
$$

is $J$-plurisubharmonic on $U$ for every $q \in V$. By assumption the function $u-c|z|^{2}$ is $J$-plurisubharmonic on $D \cap U$. Set $\tau=2 B / c$ and define, for every point $q \in V$, the function $\Psi_{q}$ by

$$
\Psi_{q}(z)= \begin{cases}\theta_{r}\left(|z-z(q)|^{2}\right) \exp \left(\theta_{r}(A|z-z(q)|)\right) \exp (\tau u(z)) & \text { if } z \in D \cap U, \\ \exp (1+\tau u) & \text { on } D \backslash U .\end{cases}
$$

Then for every $0<\varepsilon \leq B$, the function $\log \left(\Psi_{q}\right)-\varepsilon|z|^{2}$ is $J$-plurisubharmonic on $D \cap U$ and hence $\Psi_{q}$ is $J$-plurisubharmonic on $D \cap U$. Since $\Psi_{q}$ coincides with $\exp (\tau u)$ outside $U$, it is globally $J$-plurisubharmonic on $D$.

Let $f \in \mathcal{O}_{J}(\Delta, D)$ be such that $f(0)=q \in V_{1}$ and $(\partial f / \partial x)(0)=v / \alpha$ where $v \in T_{q} M$ and $\alpha>0$. For $\zeta$ sufficiently close to 0 we have

$$
f(\zeta)=q+\mathrm{d} f(0)(\zeta)+\mathcal{O}\left(|\zeta|^{2}\right) .
$$

Setting $\zeta=\zeta_{1}+i \zeta_{2}$ and using the $J$-holomorphy condition $\mathrm{d} f(0) \circ J_{\mathrm{st}}=$ $J \circ \mathrm{d} f(0)$, we may write

$$
\mathrm{d} f(0)(\zeta)=\zeta_{1} \mathrm{~d} f(0)(\partial / \partial x)+\zeta_{2} J(\mathrm{~d} f(0)(\partial / \partial x)) .
$$

Consider the function

$$
\varphi(\zeta)=\frac{\Psi_{q}(f(\zeta))}{|\zeta|^{2}}
$$

BULLETIN DE LA SOCIÉTÉ MATHÉMATIQUE DE FRANCE 
which is subharmonic on $\Delta \backslash\{0\}$. Since

$$
\varphi(\zeta)=\frac{|f(\zeta)-q|^{2}}{|\zeta|^{2}} \exp (A|f(\zeta)-q|) \exp (\tau u(f(\zeta)))
$$

for $\zeta$ close to 0 and

$$
|\mathrm{d} f(0)(\zeta)| \leq|\zeta|(\|I+J\| \cdot\|\mathrm{d} f(0)(\partial / \partial x)\|)
$$

we obtain that $\lim \sup _{\zeta \rightarrow 0} \varphi(\zeta)$ is finite. Moreover setting $\zeta_{2}=0$ we have

$$
\limsup _{\zeta \rightarrow 0} \varphi(\zeta) \geq\|\mathrm{d} f(0)(\partial / \partial x)\|^{2} \exp (-2 B|u(q)| / c) .
$$

Applying the maximum principle to a subharmonic extension of $\varphi$ on $\Delta$ we obtain the inequality

$$
\|\mathrm{d} f(0)(\partial / \partial x)\|^{2} \leq \exp (1+2 B|u(q)| / c) .
$$

Hence, by definition of the Kobayashi-Royden infinitesimal pseudometric, we obtain for every $q \in D \cap V_{1}, v \in T_{q} M$ :

$$
K_{(D, J)}(q, v) \geq\left[\exp \left(-1-2 B \frac{|u(q)|}{c}\right)\right]^{1 / 2}\|v\| .
$$

Consider now the Kobayashi ball $B_{(D, J)}(q, \alpha)=\left\{w \in D ; d_{(D, J)}^{K}(w, q)<\alpha\right\}$. It follows from Lemma 2.2 of [3] (whose proof is identical in the almost complex setting) that there is a neighborhood $V$ of $p$, relatively compact in $V_{1}$ and a positive constant $s<1$, independent of $q$, such that for every $f \in \mathcal{O}_{J}(\Delta, D)$ satisfying $f(0) \in D \cap V$ we have $f(s \Delta) \subset D \cap U$. This gives the inequality (1).

\section{Scaling and estimates of the Kobayashi-Royden metric}

In this section we present a precise lower estimate on the Kobayashi-Royden infinitesimal metric on a strictly pseudoconvex domain in $(M, J)$.

Theorem 1. - Let $M$ be a real $2 n$-dimensional manifold with an almost complex structure $J$ and let $D=\{\rho<0\}$ be a relatively compact domain in $(M, J)$. We assume that $\rho$ is a $\mathcal{C}^{2}$ defining function of $D$, strictly $J$-plurisubharmonic in a neighborhood of $\bar{D}$. Then there exists a positive constant $c$ such that

$$
K_{(D, J)}(p, v) \geq c\left[\frac{\left|\partial_{J} \rho(p)(v-i J(p) v)\right|^{2}}{|\rho(p)|^{2}}+\frac{\|v\|^{2}}{|\rho(p)|}\right]^{1 / 2},
$$

for every $p \in D$ and every $v \in T_{p} M$.

We start with the small almost complex deformations of the standard structure. In the second subsection, we consider the case of an arbitrary almost complex structure, not necessarily close to the standard one. We use non-isotropic dilations in special coordinates "reducing" an almost complex structure in order 
to represent a strictly pseudoconvex hypersurface on an almost complex manifold as the Siegel sphere equipped with an arbitrary small deformation of the standard structure. We stress that such a representation cannot be obtained by the isotropic dilations of Lemma 1 since the limit hypersurface is just a hyperplane.

4.1. Small deformations of the standard structure. - We start the proof of Theorem 1 with the following:

Proposition 4. - Let $D=\{\rho<0\}$ be a bounded domain in $\mathbb{C}^{n}$, where $\rho$ is a $\mathcal{C}^{2}$ defining function of $D$, strictly $J_{\text {st }}$-plurisubharmonic in a neighborhood of $\bar{D}$. Then there exist positive constants $c$ and $\lambda_{0}$ such that for every almost complex structure $J$ defined in a neighborhood of $\bar{D}$ and such that $\left\|J-J_{\mathrm{st}}\right\|_{\mathcal{C}^{2}(\bar{D})} \leq \lambda_{0}$ estimate (3) is satisfied for every $p \in D, v \in \mathbb{C}^{n}$.

Proof. - We note that according to Proposition 3 (see estimate (2)) it is sufficient to prove the inequality near $\partial D$. Suppose by contradiction that there exists a sequence $\left(p^{\nu}\right)$ of points in $D$ converging to a boundary point $q$, a sequence $\left(v^{\nu}\right)$ of unitary vectors and a sequence $\left(J_{\nu}\right)$ of almost complex structures defined in a neighborhood of $\bar{D}$, satisfying $\lim _{\nu \rightarrow \infty}\left\|J_{\nu}-J_{\text {st }}\right\|_{\mathcal{C}^{2}(\bar{D})}=0$, such that the quotient

$$
K_{\left(D \cap U, J_{\nu}\right)}\left(p^{\nu}, v^{\nu}\right)\left[\frac{\left|\partial_{J_{\nu}} \rho\left(p^{\nu}\right)\left(v^{\nu}-i J_{\nu}\left(p^{\nu}\right) v^{\nu}\right)\right|^{2}}{\left|\rho\left(p^{\nu}\right)\right|^{2}}+\frac{\left\|v^{\nu}\right\|^{2}}{\left|\rho\left(p^{\nu}\right)\right|}\right]^{-1 / 2}
$$

tends to 0 as $\nu$ tends to $\infty$, where $U$ is a neighborhood of $q$. For sufficiently large $\nu$ denote by $\delta_{\nu}$ the euclidean distance from $p^{\nu}$ to the boundary of $D$ and by $q^{\nu} \in \partial D$ the unique point such that $\left|p^{\nu}-q^{\nu}\right|=\delta_{\nu}$. Without loss of generality we assume that $q=0$, that $T_{0}(\partial D)=\left\{z:=\left(z^{\prime}, z_{n}\right) \in \mathbb{C}^{n} ; \operatorname{Re}\left(z_{n}\right)=0\right\}$ and that $J_{\nu}\left(q^{\nu}\right)=J_{\text {st }}$ for every $\nu$.

Consider a sequence of biholomorphic (for the standard structure) transformations $T^{\nu}$ in a neigborhood of the origin, such that $T^{\nu}\left(q^{\nu}\right)=0$ and such that the image $D^{\nu}:=T^{\nu}(D)$ satisfies

$$
T_{0}\left(\partial D^{\nu}\right)=\left\{z \in \mathbb{C}^{n} ; \operatorname{Re}\left(z_{n}\right)=0\right\} .
$$

We point out that the sequence $\left(T^{\nu}\right)_{\nu}$ converges uniformly to the identity map since $q^{\nu} \rightarrow q=0$ as $\nu \rightarrow \infty$ and hence that the sequence $\left(\left(T^{\nu}\right)^{-1}\right)_{\nu}$ is bounded. We still denote by $J_{\nu}$ the direct image $\left(T^{\nu}\right)_{*}\left(J_{\nu}\right)$. Let $U_{1}$ be a neighborhood of the origin such that $\bar{U} \subset U_{1}$. For sufficiently large $\nu$ we have $T^{\nu}(U) \subset U_{1}$. We may assume that every domain $D^{\nu}$ is defined on $U_{1}$ by

$$
D^{\nu} \cap U_{1}=\left\{z \in U_{1} ; \rho^{\nu}(z):=\operatorname{Re}\left(z_{n}\right)+\left|z^{\prime}\right|^{2}+\mathcal{O}\left(|z|^{3}\right)<0\right\},
$$


and that the sequence $\left(\widehat{p}^{\nu}=T^{\nu}\left(p^{\nu}\right)=\left(0^{\prime},-\delta_{\nu}\right)\right)_{\nu}$ is on the real inward normal to $\partial D^{\nu}$ at 0 . Of course, the functions $\rho^{\nu}$ converge uniformly with all derivatives to the defining function $\rho$ of $D$. In what follows we omit the hat and write $p^{\nu}$ instead of $\widehat{p}^{\nu}$.

Denote by $R$ the function

$$
R(z)=\operatorname{Re}\left(z_{n}\right)+\left|z^{\prime}\right|^{2}+\left(\operatorname{Re}\left(z_{n}\right)+\left|z^{\prime}\right|^{2}\right)^{2} .
$$

There is a neighborhood $V_{0}$ of the origin in $\mathbb{C}^{n}$ such that the function $R$ is strictly $J_{\text {st }}$-plurisubharmonic on $V_{0}$. Fix $\alpha>0$ small enough such that the point $z^{\alpha}=\left(0^{\prime},-\alpha\right)$ belongs to $V_{0}$. Consider the dilation $\Lambda_{\nu}$ defined on $\mathbb{C}^{n}$ by $\Lambda_{\nu}(z)=\left(\left(\alpha / \delta_{\nu}\right)^{1 / 2} z^{\prime},\left(\alpha / \delta_{\nu}\right) z_{n}\right)$. If we set $J^{\nu}:=\Lambda_{\nu} \circ J_{\nu} \circ\left(\Lambda_{\nu}\right)^{-1}$ then we have:

Lemma 5. - $\lim _{\nu \rightarrow \infty} J^{\nu}=J_{\mathrm{st}}$, uniformly on compact subsets of $\mathbb{C}^{n}$.

Proof. - Considering $J$ as a matrix valued function, we may assume that the Taylor expansion of $J_{\nu}$ at the origin is given by $J_{\nu}=J_{\mathrm{st}}+L_{\nu}(z)+\mathcal{O}\left(|z|^{2}\right)$ on $U$, uniformly with respect to $\nu$. Hence

$$
J^{\nu}\left(z^{0}\right)(v)=J_{\mathrm{st}}(v)+L_{\nu}\left(z^{\prime},\left(\delta_{\nu} / \alpha\right)^{1 / 2} z_{n}\right)(v)+\mathcal{O}\left(\mid\left(\delta_{\nu} \mid\right)\|v\| .\right.
$$

Since $\lim _{\nu \rightarrow \infty} L_{\nu}=0$ by assumption, we obtain the desired result.

Let $\widetilde{\rho}^{\nu}:=\left(\alpha / \delta_{\nu}\right) \rho^{\nu} \circ \Lambda_{\nu}^{-1}$ and $G^{\nu}:=\left\{z \in \Lambda_{\nu}\left(U_{1}\right) ; \widetilde{\rho}^{\nu}(z)<0\right\}$. Then the function $R^{\nu}:=\widetilde{\rho}^{\nu}+\left(\widetilde{\rho}^{\nu}\right)^{2}$ converges with all its derivatives to $R$, uniformly on compact subsets of $\mathbb{C}^{n}$. Hence $R^{\nu}$ is strictly plurisubharmonic on $V_{0}$ and according to Lemma 5 there is a positive constant $C$ such that for sufficiently large $\nu$ the function $R^{\nu}-C|z|^{2}$ is strictly $J^{\nu}$-plurisubharmonic on $V_{0}$. Since $\sup _{z \in G^{\nu} \cap \partial V_{0}}\left(R^{\nu}(z)-C|z|^{2}\right)=-C^{\prime}<0$, the function

$$
\widetilde{R}^{\nu}:= \begin{cases}R^{\nu}-C|z|^{2} & \text { on } D^{\nu} \cap V_{0}, \\ -\frac{1}{2} C^{\prime} & \text { on } D^{\nu} \backslash V_{0},\end{cases}
$$

is $J^{\nu}$-plurisubharmonic on $G^{\nu}$, strictly $J^{\nu}$-plurisubharmonic on $G^{\nu} \cap V_{0}$. Since $z^{\alpha}$ belongs to $V_{0}$, it follows from the Proposition 3 (see estimate (2)) that there exists a positive constant $C^{\prime}>0$ such that for sufficiently large $\nu$ we have for every $v \in \mathbb{C}^{n}$,

$$
K_{\left(G^{\nu}, J^{\nu}\right)}\left(z^{\alpha}, v\right) \geq C^{\prime}\|v\| .
$$

Moreover for $v \in \mathbb{C}^{n}$ and for sufficiently large $\nu$ we have:

$$
K_{\left(D^{\nu} \cap U_{1}, J_{\nu}\right)}\left(p^{\nu}, v\right)=K_{\left(G^{\nu}, J^{\nu}\right)}\left(z^{\alpha}, \Lambda_{\nu}(v)\right) \geq C^{\prime}\left\|\Lambda_{\nu}(v)\right\| .
$$

This gives the inequality

$$
K_{\left(D^{\nu} \cap U, J_{\nu}\right)}\left(p^{\nu}, v\right) \geq C^{\prime}\left(\frac{\alpha\left|v_{1}\right|^{2}}{\delta_{\nu}}+\cdots+\frac{\alpha\left|v_{n-1}\right|^{2}}{\delta_{\nu}}+\frac{\alpha^{2}\left|v_{n}\right|^{2}}{\delta_{\nu}^{2}}\right)^{1 / 2} .
$$

TOME $133-2005-\mathrm{N}^{\mathrm{O}} 2$ 
Since $C_{1} \delta_{\nu}$ is equivalent to $\left|\rho\left(p^{\nu}\right)\right|$ as $\nu \rightarrow \infty$, we obtain that there is a positive constant $C^{\prime \prime}$ such that

$$
K_{\left(D^{\nu} \cap U, J_{\nu}\right)}\left(p^{\nu}, v\right) \geq C^{\prime \prime}\left(\frac{\|v\|^{2}}{\left|\rho\left(p^{\nu}\right)\right|}+\frac{\left|v_{n}\right|^{2}}{\left|\rho\left(p^{\nu}\right)\right|^{2}}\right)^{1 / 2} .
$$

Since $J_{\nu}(0)=J_{\text {st }}$, we have

$$
\left|\partial \rho\left(p^{\nu}\right)\left(v-i J_{\nu}\left(p^{\nu}\right)(v)\right)\right|^{2}=\left|\partial_{J_{\mathrm{st}}} \rho\left(p^{\nu}\right)(v)\right|^{2}+\mathcal{O}\left(\delta_{\nu}\right)\|v\|^{2}=\left|v_{n}\right|^{2}+\mathcal{O}\left(\delta_{\nu}\right)\|v\|^{2} .
$$

Hence there exists a positive constant $\widetilde{C}$ such that

$$
K_{\left(D^{\nu} \cap U, J_{\nu}\right)}\left(p^{\nu}, v\right) \geq \widetilde{C}\left(\frac{\|v\|^{2}}{\left|\rho\left(p^{\nu}\right)\right|}+\frac{\left|\partial_{J} \rho\left(p^{\nu}\right)\left(v-i J_{\nu}\left(p^{\nu}\right)(v)\right)\right|^{2}}{\left|\rho\left(p^{\nu}\right)\right|^{2}}\right)^{1 / 2},
$$

contradicting the assumption on the quotient (4). This proves the desired estimate.

We have the following corollary:

Corollary 1. - Let $(M, J)$ be an almost complex manifold. Then every $p \in$ $M$ has a basis of complete hyperbolic neighborhoods.

Proof. - Let $p \in M$. According to Example 1 there exist a neighborhood $U$ of $p$ and a diffeomorphism $z: U \rightarrow \mathbb{B}$, centered at $p$, such that the function $|z|^{2}$ is strictly $J$-plurisubharmonic on $U$ and $\left\|z_{\star}(J)-J_{\text {st }}\right\|_{\mathcal{C}^{2}(U)} \leq \lambda_{0}$. Hence the open ball $\left\{x \in \mathbb{C}^{n} ;\|x\|<\frac{1}{2}\right\}$ equipped with the structure $z_{\star}(J)$ satisfies the hypothesis of Theorem 4. Now the estimate on the Kobaysahi-Royden metric given by this theorem implies that this ball is complete hyperbolic by the standard integration argument.

4.2. Arbitrary almost complex structures. - We turn now to the proof of Theorem 1 on an arbitrary strictly pseudoconvex domain in an almost complex manifold $(M, J)$ ( $J$ is not supposed to be a small deformation of the standard structure). In view of Proposition 3 it suffices to prove the statement in a neighborhood $U$ of a boundary point $q \in \partial D$. Considering local coordinates $z$ centered at $q$, we may assume that $D \cap U$ is a domain in $\mathbb{C}^{n}$ and $0 \in \partial D$, $J(0)=J_{\text {st }}$. The idea of the proof is to reduce the situation to the case of a small deformation of the standard structure considered in Proposition 4. In the case of real dimension four Theorem 1 is a direct corollary of Proposition 4 . In the case of arbitrary dimension the proof of Theorem 1 requires a slight modification of Proposition 4. So we treat this case seperately. 
4.2.1. Case where $\operatorname{dim} M=4$. - According to [16, Cor. 3.1.2], there exist a neighborhood $U$ of $q$ in $M$ and complex coordinates $z=\left(z_{1}, z_{2}\right): U \rightarrow \mathbb{B}_{2} \subset \mathbb{C}^{2}$, $z(0)=0$ such that $z_{*}(J)(0)=J_{\mathrm{st}}$ and moreover, a map $f: \Delta \rightarrow \mathbb{B}$ is $J^{\prime}:=z_{*}(J)$-holomorphic if it satisfies the equations

$$
\frac{\partial f_{j}}{\partial \bar{\zeta}}=A_{j}\left(f_{1}, f_{2}\right)\left(\frac{\overline{\partial f_{j}}}{\partial \zeta}\right), \quad j=1,2
$$

where $A_{j}(z)=O(|z|)$.

In order to obtain such coordinates, one can consider two transversal foliations of $\mathbb{B}$ by $J^{\prime}$-holomorphic curves (see [13]) and then take these curves into the lines $z_{j}=$ const by a local diffeomorphism. The direct image of the almost complex structure $J$ under such a diffeomorphism has a diagonal matrix $J^{\prime}\left(z_{1}, z_{2}\right)=\left(a_{j, k}(z)\right)_{j, k}$ with $a_{1,2}=a_{2,1}=0$ and $a_{j, j}=i+\alpha_{j, j}$ where $\alpha_{j, j}(z)=\mathcal{O}(|z|)$ for $j=1,2$. We point out that the lines $z_{j}=$ const are $J$-holomorphic after a suitable parametrization (which, in general, is not linear).

In what follows we omit the prime and denote this structure again by $J$. We may assume that the complex tangent space $T_{0}(\partial D) \cap J(0) T_{0}(\partial D)=$ $T_{0}(\partial D) \cap i T_{0}(\partial D)$ is given by $\left\{z_{2}=0\right\}$. In particular, we have the following expansion for the defining function $\rho$ of $D$ on $U$

$$
\rho(z, \bar{z})=2 \operatorname{Re}\left(z_{2}\right)+2 \operatorname{Re} K(z)+H(z)+\mathcal{O}\left(|z|^{3}\right),
$$

where $K(z)=\sum k_{\nu, \mu} z_{\nu} z_{\mu}, k_{\nu, \mu}=k_{\mu, \nu}$ and $H(z)=\sum h_{\nu, \mu} z_{\nu} \bar{z}_{\mu}, h_{\nu, \mu}=\bar{h}_{\mu, \nu}$.

Lemma 6. - The domain $D$ is strictly $J_{\mathrm{st}}$-pseudoconvex near the origin.

Proof of Lemma 6. - Consider a complex vector $v=\left(v_{1}, 0\right)$ tangent to $\partial D$ at the origin. Let $f: \Delta \rightarrow \mathbb{C}^{2}$ be a $J$-holomorphic disc centered at the origin and tangent to $v: f(\zeta)=v \zeta+\mathcal{O}\left(|\zeta|^{2}\right)$. Since $A_{2}=\mathcal{O}(|z|)$, it follows from the $J$ holomorphy equation $(5)$ that $\left(f_{2}\right)_{\zeta \bar{\zeta}}(0)=0$. This implies $(\rho \circ f)_{\zeta \bar{\zeta}}(0)=H(v)$. Thus, the Levi form with respect to $J$ coincides with the Levi form with respect to $J_{\text {st }}$ on the complex tangent space of $\partial D$ at the origin.

Consider the non-isotropic dilations $\Lambda_{\delta}:\left(z_{1}, z_{2}\right) \mapsto\left(\delta^{-1 / 2} z_{1}, \delta^{-1} z_{2}\right)=\left(w_{1}, w_{2}\right)$ with $\delta>0$. If $J$ has the above diagonal form in the coordinates $\left(z_{1}, z_{2}\right)$ in $\mathbb{C}^{2}$, then its direct image $J_{\delta}=\left(\Lambda_{\delta}\right)_{*}(J)$ has the form $J_{\delta}\left(w_{1}, w_{2}\right)=$ $\left(a_{j k}\left(\delta^{1 / 2} w_{1}, \delta w_{2}\right)\right)_{j k}$ and so $J_{\delta}$ tends to $J_{\text {st }}$ in the $\mathcal{C}^{2}$ norm as $\delta \rightarrow 0$. On the other hand, $\partial D$ is, in the coordinates $w$, the zero set of the function $\rho_{\delta}=\delta^{-1}\left(\rho \circ \Lambda_{\delta}^{-1}\right)$. As $\delta \rightarrow 0$, the function $\rho_{\delta}$ tends to the function $2 \operatorname{Re} w_{2}+2 \operatorname{Re} K\left(w_{1}, 0\right)+H\left(w_{1}, 0\right)$ which defines a $J_{\text {st }}$-strictly pseudoconvex domain by Lemma 6 . So we may apply Proposition 4 . This proves Theorem 1 in dimension 4 . 
4.2.2. Case where $\operatorname{dim} M=2 n$. - In this case Proposition 4 is not directly applicable since $J$ can not be deformed by the non-isotropic dilations to the standard structure. Instead we use the invariance of the Levi form with respect to the non-isotropic dilations.

We suppose that in a neighborhhod of the origin we have $J=J_{\text {st }}+\mathcal{O}(|z|)$. We also may assume that in these coordinates the defining function $\rho$ of $D$ has the form $\rho=2 \operatorname{Re} z_{n}+2 \operatorname{Re} K(z)+H(z)+\mathcal{O}\left(|z|^{3}\right)$, where $K$ and $H$ are defined similarly to the 4 -dimensional case and $\rho$ is strictly $J$-plurisubharmonic at the origin. We use the notation $z=\left(z^{\prime}, z_{n}\right)$.

Consider the non-isotropic dilations $\Lambda_{\delta}:\left(z^{\prime}, z_{n}\right) \mapsto\left(w^{\prime}, w_{n}\right)=\left(\delta^{-1 / 2} z^{\prime}, \delta^{-1} z_{n}\right)$ and set $J_{\delta}=\left(\Lambda_{\delta}\right)_{*}(J)$. Then $J_{\delta}$ tends to the almost complex structure $J_{0}(z)=J_{\text {st }}+L\left(z^{\prime}, 0\right)$ where $L\left(z^{\prime}, 0\right)=\left(L_{k j}\left(z^{\prime}, 0\right)\right)_{k j}$ denotes a matrix with $L_{k, j}=0$ for $k=1, \ldots, n-1, j=1, \ldots, n, L_{n, n}=0$ and $L_{n, j}\left(z^{\prime}, 0\right)$, $j=1, \ldots, n-1$ being (real) linear forms in $z^{\prime}$.

Let $\rho_{\delta}=\delta^{-1}\left(\rho \circ \Lambda_{\delta}^{-1}\right)$. As $\delta \rightarrow 0$, the function $\rho_{\delta}$ tends to the function $\widetilde{\rho}=2 \operatorname{Re} z_{n}+2 \operatorname{Re} K\left(z^{\prime}, 0\right)+H\left(z^{\prime}, 0\right)$ in the $\mathcal{C}^{2}$ norm. By the invariance of the Levi form we have $\mathcal{L}^{J}(\rho)(0)\left(\Lambda_{\delta}^{-1}(v)\right)=\mathcal{L}^{J_{\delta}}\left(\rho \circ \Lambda_{\delta}^{-1}\right)(0)(v)$. Since $\rho$ is strictly $J$-plurisubharmonic, multiplying by $\delta^{-1}$ and passing to the limit at the right side as $\delta \rightarrow 0$, we obtain that $\mathcal{L}^{J_{0}}(\widetilde{\rho})(0)(v) \geq 0$ for any $v$. Now let $v=\left(v^{\prime}, 0\right)$. Then $\Lambda_{\delta}^{-1}(v)=\delta^{1 / 2} v$ and so $\mathcal{L}^{J}(\rho)(0)(v)=\mathcal{L}^{J_{\delta}}\left(\rho_{\delta}\right)(0)(v)$. Passing to the limit as $\delta$ tends to zero, we obtain that $\mathcal{L}^{J_{0}}(\tilde{\rho})(0)(v)>0$ for any $v=\left(v^{\prime}, 0\right)$ with $v^{\prime} \neq 0$.

Consider now the function $R=\widetilde{\rho}+\widetilde{\rho}^{2}$. Then

$$
\mathcal{L}^{J_{0}}(R)(0)(v)=\mathcal{L}^{J_{0}}(\widetilde{\rho})(0)(v)+2 v_{n} \bar{v}_{n},
$$

so $R$ is strictly $J_{0}$-plurisubharmonic in a neighborhood of the origin. Thus the functions $R^{\nu}$ used in the proof of Proposition 4 are strictly $J^{\nu}$-plurisubharmonic and their Levi forms are bounded from below by a positive constant independent of $\nu$. This allows to use Proposition 3 and the proof can be proceeded quite similarly to the proof of Proposition 4 without any changes.

REMARK. - As it was brought to our attention by J.-P. Rosay, our proof of Theorem 1 also gives the following local version: If $D$ is an arbitrary domain (not necessarily relatively compact) in an almost complex manifold $(M, J)$, strictly pseudoconvex at a boundary point $q$, then the estimate (3) still holds for any point $p \in D$ in a neighborhood of $q$. 


\section{BIBLIOGRAPHY}

[1] Berteloot (F.) - Attraction des disques analytiques et continuité höldérienne d'applications holomorphes propres, in Topics in complex analysis (Warsaw, 1992), Banach Center Publ., vol. 31, Polish Acad. Sci., Warsaw, 1995, pp. 91-98.

[2] _ Principe de Bloch et estimations de la métrique de Kobayashi des domaines de $\mathbb{C}^{2}$, J. Geom. Anal., t. 13 (2003), pp. 29-37.

[3] Chirka (E.M.), Coupet (B.) \& Sukhov (A.B.) - On boundary regularity of analytic discs, Michigan Math. J., t. 46 (1999), pp. 271-279.

[4] Debalme (R.) - Kobayashi hyperbolicity of almost complex manifolds, Preprint IRMA, arXiv: math.CV/9805130, 1999.

[5] Debalme (R.) \& Ivashkovich (S.) - Complete hyperbolic neighborhoods in almost-complex surfaces, Internat. J. Math., t. 12 (2001), pp. 211-221.

[6] Diederich (K.) \& Fornæss (J.E.) - Proper holomorphic maps onto pseudoconvex domains with real-analytic boundary, Ann. of Math. (2), t. 110 (1979), pp. 575-592.

[7] Graham (I.) - Boundary behavior of the Carathéodory and Kobayashi metrics on strongly pseudoconvex domains in $\mathbb{C}^{n}$ with smooth boundary, Trans. Amer. Math. Soc., t. 207 (1975), pp. 219-240.

[8] HagGui (F.) - Fonctions FSH sur une variété presque complexe, C. R. Math. Acad. Sci. Paris, t. 335 (2002), pp. 509-514.

[9] Ivashkovich (S.) \& Rosay (J.-P.) - Schwarz-type lemmas for solutions of $\bar{\partial}$-inequalities and complete hyperbolicity of almost complex manifolds, arXiv: math. CV/0310474, 2003.

[10] Kerzman (N.) \& Rosay (J.-P.) - Fonctions plurisousharmoniques d'exhaustion bornées et domaines taut, Math. Ann., t. 257 (1981), pp. 171184.

[11] Kobayashi (S.) - Almost complex manifolds and hyperbolicity, Results Math., t. 40 (2001), pp. 246-256, Dedicated to Shiing-Shen Chern on his 90th birthday.

[12] Kruglikov (B.S.) - Existence of close pseudoholomorphic disks for almost complex manifolds and their application to the Kobayashi-Royden pseudonorm, Funktsional. Anal. i Prilozhen., t. 33 (1999), pp. 46-58, 96.

[13] Nijenhuis (A.) \& Woolf (W.B.) - Some integration problems in almost-complex and complex manifolds, Ann. of Math. (2), t. 77 (1963), pp. 424-489.

[14] Pinchuk (S.) - The scaling method and holomorphic mappings, in Several complex variables and complex geometry, Part 1 (Santa Cruz, CA, 1989), Proc. Sympos. Pure Math., vol.52, Amer. Math. Soc., Providence, RI, 1991, pp. 151-161. 
[15] Sibony (N.) - A class of hyperbolic manifolds, in Recent developments in several complex variables (Proc. Conf., Princeton Univ., Princeton, N.J., 1979), Ann. of Math. Stud., vol. 100, Princeton Univ. Press, Princeton, N.J., 1981, pp. 357-372.

[16] Sikorav (J.-C.) - Some properties of holomorphic curves in almost complex manifolds, in Holomorphic curves in symplectic geometry, Progr. Math., vol. 117, Birkhäuser, Basel, 1994, pp. 165-189. 